\title{
Shape Memory Photonic Metamaterial
}

\author{
Masanori Tsuruta $^{1,2}$, João Valente ${ }^{1}$, Behrad Gholipour ${ }^{1,3}$, Kevin F. MacDonald ${ }^{1}$, Eric Plum ${ }^{1}$, \\ and Nikolay I. Zheludev ${ }^{1,3}$ \\ ${ }^{1}$ Optoelectronics Research Centre and Centre for Photonic Metamaterials, \\ University of Southampton, SO17 1BJ, UK \\ ${ }^{2}$ Energy and Environment $R \& D$ Center, Asahi Kasei, Japan \\ ${ }^{3}$ Centre for Disruptive Photonic Technologies and The Photonics Institute, \\ Nanyang Technological University, Singapore 637371, Singapore \\ Authore-mail address: erp@orc.soton.ac.uk, niz@orc.soton.ac.uk
}

\begin{abstract}
We report the first reconfigurable metamaterial based on the shape memory alloy. In the heating cycle structural elements of this metamaterials exhibit a hysteresis-type shape transformation that leads to non-volatile switching of its plasmonic properties.

OCIS codes: (160.3918) Metamaterials; (250.6715) Switching.
\end{abstract}

\section{Introduction}

Mechanically reconfigurable metamaterials actuated by thermal, electrical, magnetic or optical signals enable fast and high-contrast modulation of light. However, existing reconfigurable nanomembrane metamaterials do not offer a rewritable memory functionality, i.e. their optical properties only depend on the applied control signal that drives actuation, but not on its history. Mechanically reconfigurable nanostructures exploit that the optical properties of metamaterials are sensitive to nanoscale movements of their components. Therefore, shape memory alloys, which recover their original shape upon heating after deformation, provide an opportunity to realize memory metamaterials. Here we fabricate the first reconfigurable metamaterial based on a shape memory alloy, demonstrating a memory metamaterial, which exhibits different transmission levels when heated or cooled to a given temperature.

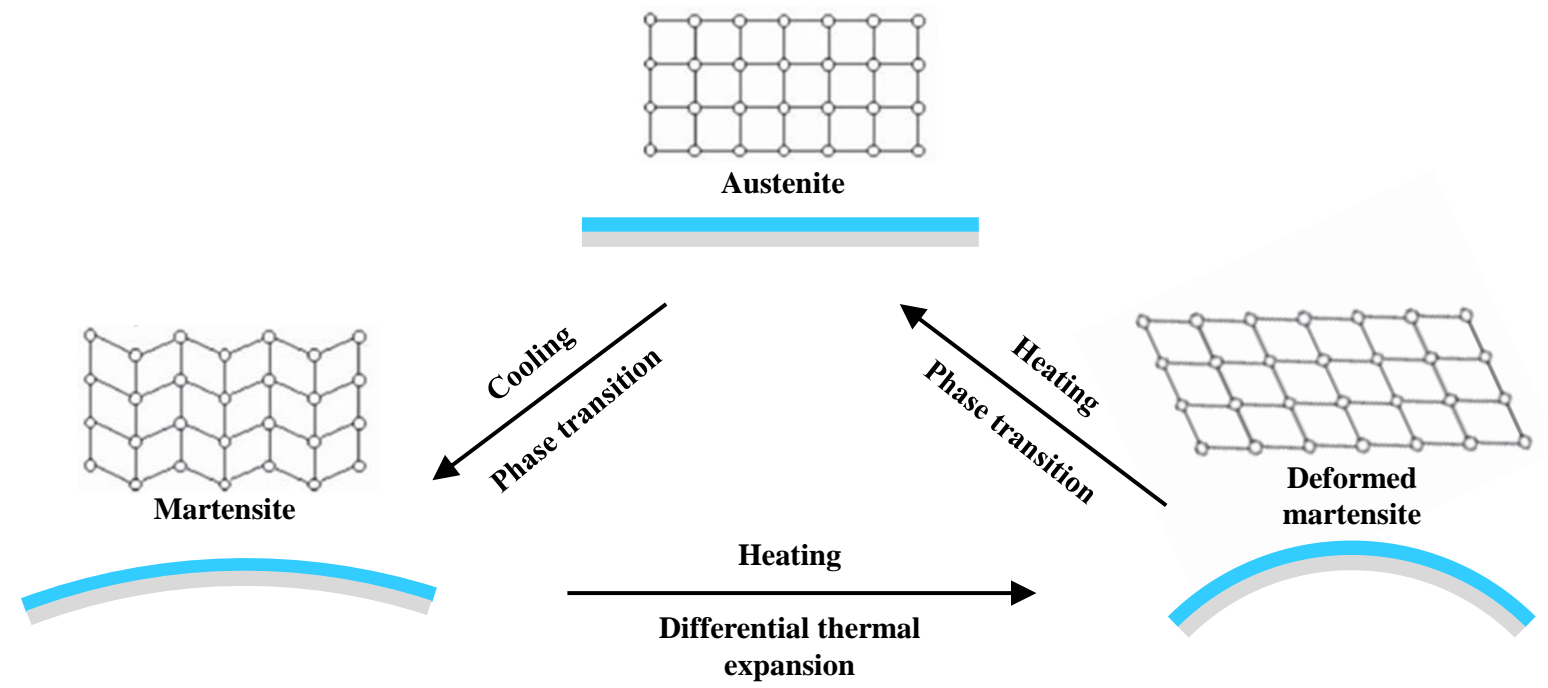

Fig. 1. Schematic operating principle of shape memory metamaterial. A bimorph beam consisting of shape memory alloy (blue) and dielectric (gray) will deform upon heating due to differential thermal expansion. Further heating causes shape recovery by engaging the martensite to austenite phase transition. Cooling will return the structure to its original state with hysteresis by engaging the austenite to martensite phase transition at a lower temperature. 


\section{Results}

The shape memory metamaterial was manufactured by DC magnetron cosputtering of a $50 \mathrm{~nm}$ layer of CuAlNi shape memory alloy on a silicon nitride membrane of $50 \mathrm{~nm}$ thickness. In order to realize a plasmonic reconfigurable metamaterial, an additional layer of $50 \mathrm{~nm}$ of gold was deposited on top of the shape memory alloy to provide plasmonic properties. The metamaterial was created by focused ion beam milling, cutting through the gold, shape memory alloy and silicon nitride layers to fabricate an array of freestanding nanostructured bridge actuators of about $30 \mu \mathrm{m}$ length. Actuation of the nanostructure is driven by temperature changes due to differential thermal expansion of the constituent materials as well as phase transitions of the shape memory alloy. Fig. 1 schematically illustrates the operating principle of the shape memory metamaterial shown by Fig. 2a, where actuation of a single simplified bridge beam actuator is shown to illustrate the concept.

The temperature-dependent optical properties of the shape memory metamaterial are illustrated by Figs. $2 \mathrm{~b}$ and $2 \mathrm{c}$ for transmission of light polarized perpendicular to the bridges. The metamaterial's transmission has a strong temperature dependence with hysteresis which is illustrated by Fig. $2 \mathrm{c}$ for the telecommunications wavelength of $1300 \mathrm{~nm}$. The shape memory metamaterial is up to $14 \%$ more transparent after cooling to $150{ }^{\circ} \mathrm{C}$ compared to having been heated to $150^{\circ} \mathrm{C}$.

(a)

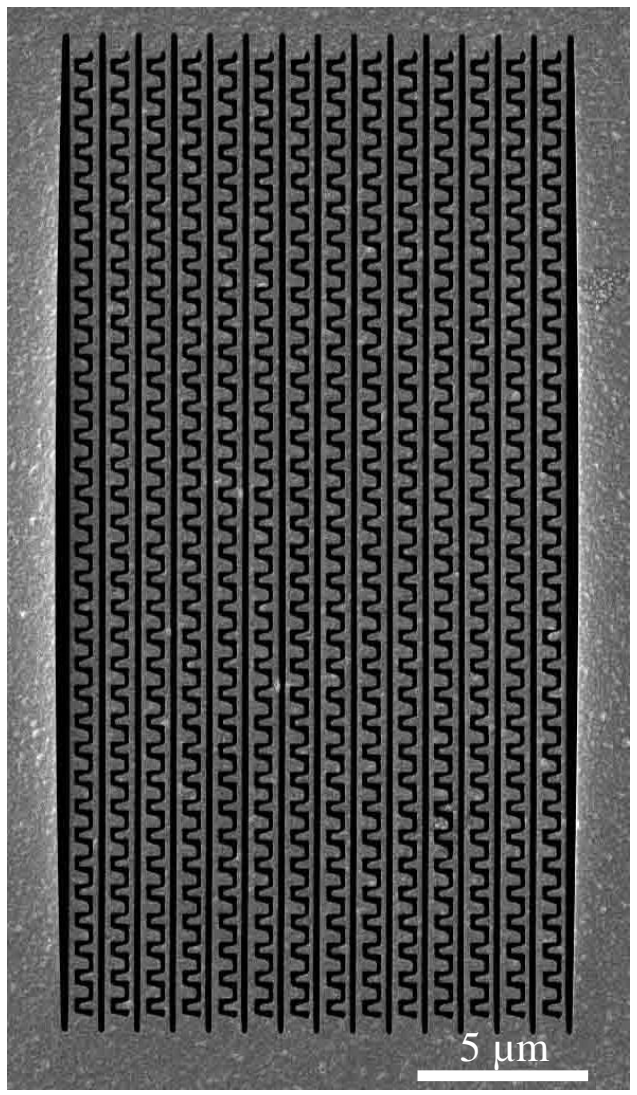

(b)

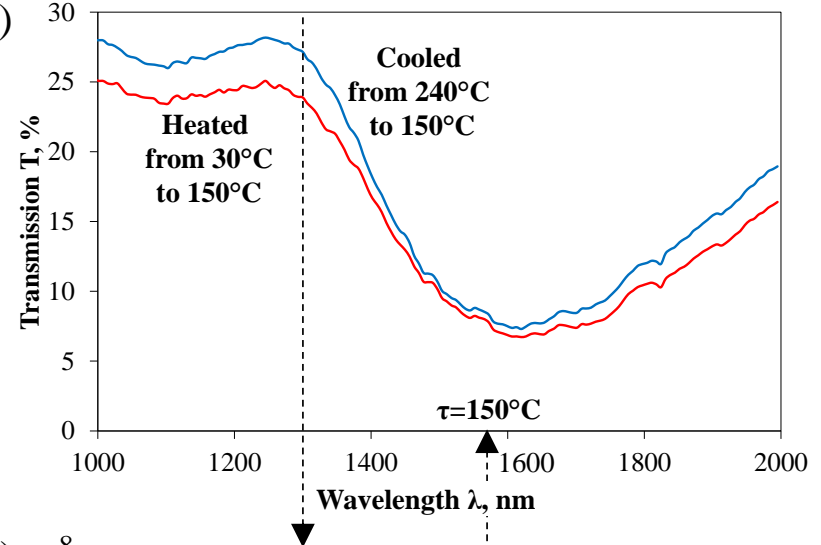

(c)

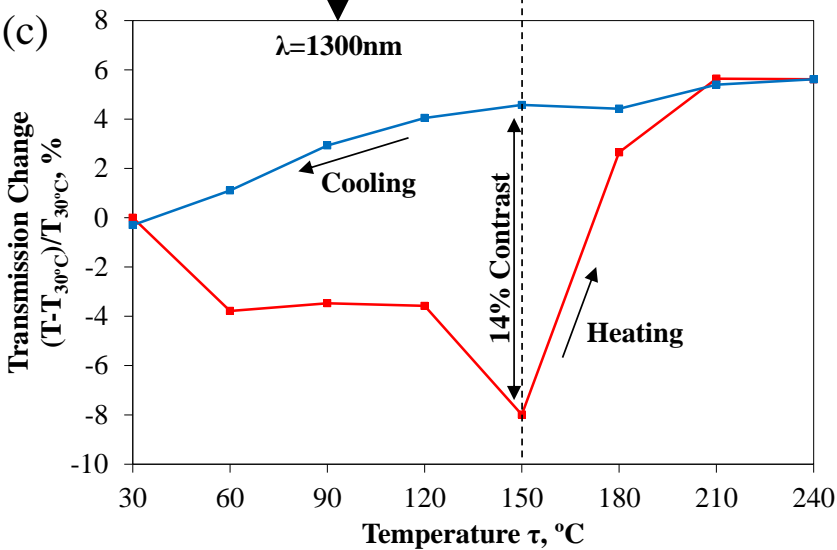

Fig. 2. Memory metamaterial. (a) Reconfigurable metamaterial consisting of $50 \mathrm{~nm}$ layers of gold, CuAlNi shape memory alloy and silicon nitride. (b) Transmission spectra after heating and cooling to $150^{\circ} \mathrm{C}$. (c) Temperature-dependent hysteresis of metamaterial transmission at a wavelength of $1300 \mathrm{~nm}$. The temperature-dependent transmission change is shown relative to a reference temperature of $30^{\circ} \mathrm{C}$.

\section{Summary}

In summary, we demonstrate the first reconfigurable metamaterial consisting of a shape memory alloy. By introducing a phase change material to mechanically reconfigurable metamaterials, we realize the first mechanically reconfigurable metamaterial with a memory functionality. We expect that shape memory metamaterials will enable advanced photonic functionalities: In principle, they can be programmed by permanent deformation into complex shapes, tuned by reversible actuation driven by thermal, electrical, magnetic or optical control signals, and reset with heat. 